\title{
Adaptive learning in agents behaviour: A framework for electricity markets simulation
}

\author{
Tiago Pinto ${ }^{\mathrm{a}}$, Zita Vale ${ }^{\mathrm{a}, *}$, Tiago M. Sousa ${ }^{\mathrm{a}}$, Isabel Praça ${ }^{\mathrm{a}}$, Gabriel Santos ${ }^{\mathrm{a}}$ and Hugo Morais ${ }^{\mathrm{b}}$ a $_{G E C A D-}$ \\ Knowledge Engineering and Decision Support Research Centre, Polytechnic of Porto, Porto, Portugal \\ ${ }^{\mathrm{b}}$ AUTomation and Control Group, Technical University of Denmark, Copenhagen, Danmark
}

\begin{abstract}
Electricity markets are complex environments, involving a large number of different entities, playing in a dynamic scene to obtain the best advantages and profits. MASCEM (Multi-Agent System for Competitive Electricity Markets) is a multi- agent electricity market simulator that models market players and simulates their operation in the market. Market players are en- tities with specific characteristics and objectives, making their decisions and interacting with other players. This paper presents a methodology to provide decision support to electricity market negotiating players. This model allows integrating different strate- gic approaches for electricity market negotiations, and choosing the most appropriate one at each time, for each different negotia- tion context. This methodology is integrated in ALBidS (Adaptive Learning strategic Bidding System) - a multiagent system that provides decision support to MASCEM's negotiating agents so that they can properly achieve their goals. ALBidS uses artificial intelligence methodologies and data analysis algorithms to provide effective adaptive learning capabilities to such negotiating entities. The main contribution is provided by a methodology that combines several distinct strategies to build actions propos- als, so that the best can be chosen at each time, depending on the context and simulation circumstances. The choosing process includes reinforcement learning algorithms, a mechanism for negotiating contexts analysis, a mechanism for the management of the efficiency/effectiveness balance of the system, and a mechanism for competitor players' profiles definition.
\end{abstract}

Keywords: Adaptive learning, artificial intelligence, electricity markets, machine learning, multiagent simulation

\section{Introduction}

Electricity markets are complex environments with very particular characteristics. A critical issue regard- ing these specific characteristics concerns the constant changes they are subject to. This is a result of the elec- tricity markets' restructuring, which was performed so that the competitiveness could be increased and conse- quently instigate a decrease in electricity prices, how- ever, it also had exponential implications in the in- crease of the complexity and unpredictability in these markets scope [35].

The constant growth in markets unpredictability re- sulted in an amplified need for market intervenient entities in foreseeing market behavior. The need for un- derstanding the market mechanisms and how the in- volved players' interaction affects the outcomes of the markets, contributed to the growth of usage of simula- tion tools [7,57]. Multi-agent based software is particularly well fitted to analyze dynamic and adaptive systems with complex interactions among its constituents, such as electricity markets $[20,23,55]$.

This paper presents a model that allows integrating different strategic approaches for electricity market ne- gotiations, and choosing the most appropriate one at each time, depending on the past performance that each strategic approach has presented for each different ne- gotiation context. This adaptive learning methodology is the core of ALBidS - Adaptive Learning strategic Bidding System, a multiagent system that has been cre- ated to provide decision support to market negotiating players. This system is integrated with an electricity markets simulator - MASCEM (Multi-Agent Simula- 
tor of Competitive Electricity Markets) $[43,48]$, so that ALBidS' benefit in supporting market players' actions can be tested and validates using realistic scenarios, based on real markets' data.

The main contribution brought by ALBidS is the integration and combination of several different method- ologies based on very distinct approaches (artificial intelligence approaches [58], data mining techniqu- es [19], forecasting algorithms [1,3,59], among many others $[2,26,34])$, in order to provide alternative sug- gestions of which are the best actions for the supported player to perform. The approach chosen as the market players' actual action is selected by the employment of reinforcement learning algorithms [27], which, for each different situation, simulation circumstances and context, decide which proposed action is the one with higher possibility of achieving the most success. The evaluation of the results has to be done by comparing the profits that the supported market player is able to achieve when participating in the electricity market.

Some of the considered approaches are supported by a mechanism that defines profiles of competitor play- ers. These profiles are built accordingly to the observed past actions and reactions of competitor players, when faced with specific situations, such as success, failure, or acting in different negotiation contexts.

The system's context awareness is provided by a context analysis mechanism, which analyses the char-acteristics and particularities of the negotiation envi- ronment in each moment, and defines different con- texts, under which the circumstances aresimilar.

ALBidS is also equipped with adaptation and learning capabilities in what concerns the balance between results performance and execution time. Considering each comprised algorithm's quality of results, and the time taken to achieve such results, a fuzzy logic [53] process is used to determine in what amount each strat- egy should reduce its execution time, by adapting itself internally, or if any is taking too long to provide not so good results, and so, be excluded from the decision making process. This process makes sure that the de- cision making process does not take longer than abso- lutely necessary, depending on the time requirements of each situation.

After this introductory section, Section 2 provides a brief review of the state of the art regarding the related work. Section 3 presents the proposed methodol- ogy of ALBidS, and Section 4 demonstrates the advan- tage of using the strategies' combination provided by ALBidS, by comparing the performance of this system with the performance of two individual bidding strate- gies. Finally, Section 5 discusses the most relevant con- clusions and future work.

\section{Related work}

The optimal bidding strategies' problematic has been the focus of a wide range of research works dur- ing the last years. Most of these works address the problem from the producers' perspective, as can be seen by [16], which provides a rather complete survey on this subject. The first approaches in this field ad-dress the problem using game theory and operational research techniques. The authors in [60] have pre- sented a market participation strategic approach based on the application of the Q-Learning reinforcement learning algorithm.

The evolution of appropriate bidding strategies for each current market conditions, using genetic algorithms (GA) is proposed in [52]. Heuristic optimization, as well as other artificial intelligence based meth- ods, has been increasingly used during the last years to deal with the problem of electricity market players' strategic behavior. This type of approach allows con- sidering a larger number of involved players in larger time horizons, as well as considering the need for play- ers' strategies dynamism through evolution over time; thus making it possible to represent a more realistic modeling of the problem.

Bid prices and quantities in a competitive electricity market context are determined in [65] using two al- gorithms based on particle swarm optimization (PSO). Probabilistic estimation is used to model opponents' bidding behavior. The experimental findings show that for nonlinear cost functions, PSO solutions provide higher expected profits than marginal cost-based bid- ding. This allows following the frequently changing conditions in the successive trading sessions of a real electricity market.

Fuzzy adaptive particle swarm optimization (FAPSO) is used in [8] to determine the optimal bidding strategy for a thermal generator for each trading period in a day-ahead market. The inertia weight of the PSO algorithm is dynamically adjusted by a fuzzy evaluation. Methods for supporting players' portfolio decisions using PSO and GA, are respectively pre- sented in $[5,6,18]$.

Electricity market price forecasting is essential to support market players in their decisions, enabling ad- equate risk management. This is, however, a rather dif- ficult task, as electricity prices are dependent from a wide set of factors and evidence unusually high spikes even when compared to other commodities markets, mainly due to the electric energy non-storability in large quantities [61]. Due to their capacity to perform 
almost every complex function [12,29], artificial neural networks (ANN) have been extensively used in price forecast, e.g. the authors in [54] use a combination of ANN and fuzzy logic. A method to forecast day-ahead electricity prices based on Self-Organizing Map (SOM) ANN and Support Vector Machine (SVM) models is presented in [64]. SOM is used to automatically cluster the data according to their similarity and SVM models for regression are built on the categories clustered by SOM separately. Parameters of the SVM models are chosen by a PSO based algorithm. A hybrid method based on wavelet transform, Auto-Regressive Integrated Moving Average (ARIMA) models and Ra- dial Basis Function Neural Networks (RBFN) to fore- cast day-ahead market price is presented in [39]. This method uses PSO to optimize the network structure which adapts the RBFN to the specified training set, reducing computation complexity and avoiding over-fitting. A SVM based approach is proposed in [4] to forecast the electricity market price for hourly nego- tiating periods of the following day. A test period of three years is used in the presented case study, concern- ing the market price of the Ontario electricity market.

The large range of developed decision support mod- els reaches its highest peak of utility when combined appropriately. For this reason, several modeling and simulation tools have been developed during the last years, facilitating the integration of different models that can fruitfully come in aid of professionals that are involved in the electricity market sector [24,38].

Multiagent technology is being increasingly used to represent, model and simulate complex and dynamic environments $[15,31,36]$. The possibility of represent- ing different entities as independent software agents with their own particular behavior and objectives; and the opportunity for easily enlarging the represented models, are some of the main reasons why multia- gent technology is widely chosen as the best option for developing complex simulation tools for constantly evolving environments such as the electricity markets. Among the most relevant multiagent based simu- lators of electricity markets the following can be re-ferred: EMCAS

(Electricity Market Complex Adaptive System) [31]; AMES (Agent-based Modeling ofElec- tricity Systems)

[33]; GAPEX (Genoa Artificial Power Exchange) [15], and MASCEM (Multi-Agent Simulator for Competitive Electricity Markets) [48,62].

However, some other simulators that are not based on a multiagent architecture also present a special rel- evance: SEPIA (Simulator for Electric Power Industry Agents) [24]; Power Web [67]; and SREMS (Short - Medium run Electricity Market Simulator) [38].
MASCEM (Multi-Agent Simulator for Competitive Electricity Markets) $[48,62]$ has been firstly introduced to the scientific community in 2003 [48]. MASCEM is able to recreate a high diversity of market clearing models, based on the mechanisms used in different countries all around the world. Negotiating players in MASCEM use several decision support strategies to pursue the best possible outcomes from the mar- ket participation. The application of game theory has been proposed in [42]; a methodology based on the Q- Learning reinforcement learning algorithm, which uses the Simulated Annealing metaheuristic to accelerate the convergence process is presented in [45]; and the application of the metalearning concept has been ap-proached in [44].

Substantial work has already been done in the field of decision support to electricity market participating players. A reference platform for comparing the per-formance of different strategies is PowerTAC (Power Trading Agent Competition) [47]. PowerTAC provides a simulation server where different approaches can compete with each other. As referred in this section, many strategic approaches that aim to provide decision support to market players have been developed. How- ever, none of the proposed biding strategies has shown to be clearly better than the other. Case studies show that different strategies perform better in distinct envi- ronments and contexts. This is the reason why the work that is proposed in this paper is essential, as it provides an intelligent, adaptive, and dynamic methodology for combining a high number of different market biding strategies, from different natures and perspectives, en- abling the advantageous use of each of these strategies whenever they show to be more adequate and present the higher chance for success for the supported player.

\section{ALBidS}

Electricity market players require strategies capable of dealing with the constant market changes, allowing adaptation to the competitors' actions and reactions, in order to achieve competitive advantage in the market negotiations. For that, adequate forecast techniques are necessary to analyze the market data, namely the his- toric market prices. The way prices are predicted can be approached in several ways, namely through the use of statistical methods, data mining techniques [19], ar- tificial neural networks (ANN) [1,3,59], support vector machines (SVM) [32], or several other methods [14, $17,25]$. There is no method that can be said to be 
best for every situation, only the best for one or other particular case.

To take advantage of the best characteristics of each technique, a new system that integrates several distinct technologies and approaches has been proposed. The ALBidS decision support system is implemented as a multiagent system, and its goal is to provide decision support to an electricity market player. Although sev- eral players can use it at the same time; in this case a different instance of ALBidS is created for each differ- ent player. There is one agent performing each distinct algorithm, detaining the exclusive knowledge of its ex- ecution, this way the system can be executing all the al- gorithms in parallel, preventing as possible the degra- dation of the method's efficiency. As each agent gets its answer, resulting from its individual strategy, it sends this result to the Main Agent, which is responsible for choosing the most appropriate answer among all that it receives. This way, strategic approaches can be seen as tools that are available to the main system, and that can be used, or adapted, depending on the circumstances and the negotiation context at each time.

Strategy agents are implemented using the factory and interface software patterns [21]. These patterns al- low all the different strategies to present a single in- terface to the Main Agent, which highly facilitates the process of launching a new agent, and also the inte- gration of further strategy agents. The creation, initial- ization, responses, and update, of all strategies follow the same interaction format, through the same inter- face. l.e. the requests, responses, and interactions are the same between the Main Agent and all the strat- egy agents; only the values and parameters differ, de- pending on what the strategy of each agent requires. This way, in order to include a new strategy, it is only required to respect this interface (containing the for- mat of the interactions for the creation, initialization, responses, and update processes), and the new strat- egy is automatically recognized by the whole system. ALBidS' conceptual model is presented in Fig. 1.

From Fig. 1 it is visible how the different modules and methodologies that are part of ALBidS interact. The different strategies/methodologies for mar- ket actions' definition are executed, using their own independent inputs. These methodologies are adapted in order to cope with the requirements from the Effi- ciency/Effectiveness (2E) balance mechanism. These adaptations refer to the reduction of each strategies' execution time, or even the exclusion of some, in case of the need being more demanding. The outputs of each methodology (action proposals for the supported 


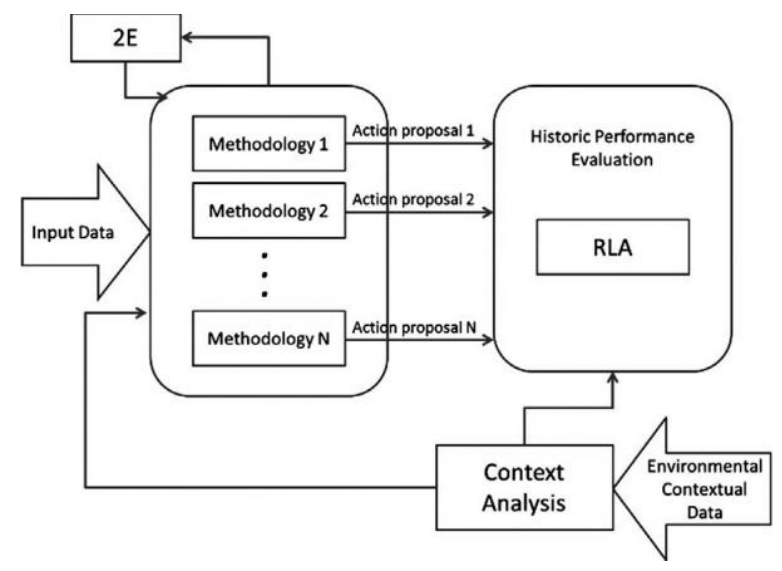

Fig. 1. ALBidS conceptual design.

market player to perform in the electricity market) are sent to the Main Agent. The Main Agent evaluates the historic performance of each strategy when acting in each different context (defined by the context anal-ysis mechanism), and chooses the action suggestion of the strategy that shows the best confidence for be- ing the most successful one. The choosing process is performed using Reinforcement Learning Algorithms (RLA).

ALBidS is connected to the MASCEM electricity markets simulator $[43,48]$, providing decision support to players negotiating in this environment. This inte- gration also provides the means for ALBidS' perfor- mance to be tested in realistic simulation scenarios. MASCEM is equipped with a data-extraction tool [49] that gathers real electricity markets data from the web- sites of several market operators, and feeds a realistic scenarios generator [56], which allow MASCEM sim- ulation scenarios to be as representative of the real- ity as possible. This way, the performance of ALBidS when supporting decisions of market players can be extrapolated to the reality, being possible to analyze the impact of the decisions in a real environment.

\subsection{Main agent}

The Main Agent interfaces the MASCEM and ALBidS systems. It receives requests from the market negotiating players of MASCEM when they require decision support, and provides them the corresponding answers. These answers are achieved after managing the ALBidS internal mechanism, including the interac- tions with the strategy agents - the agents responsible for executing the different strategies.

The choice of the most appropriate strategy to be used at each moment is based on the application of 
reinforcement learning algorithms [27]. The approach that presents the best results for a given context of the current scenario is chosen as the final response. So, given as many answers to each problem as there are algorithms, the reinforcement learning algorithm will choose the one that is most likely to present the best an- swer according to the past experience of the responses and to the present characteristics of each situation, such as the considered day, the period, and the particular market context that the algorithms are being asked to forecast.

The main reinforcement algorithm presents a distinct set of statistics for each context, which means that an algorithm that may be presenting good results for a certain period, with its output chosen more often when bidding for this context, may possibly never be chosen as the answer for another period, since they are com- pletely independent from each other. The tendencies observed when looking at the historic of negotiation periods independently from each other show that they vary much from each other, what suggests that distinct algorithms can present distinct levels of results when dealing with such different tendencies, dependant on the context.

The way the statistics are updated, and consequently the best answer chosen, can be defined by the user. ALBidS provides three reinforcement learning algo- rithms, all having in common the starting point. All the algorithms start with the same value of confidence, and then according to their particular performance that value will be updated. All algorithms also have the op- tion of being attributed a weight value that defines its importance to the system. This means that a strategy that has a higher weight value will detach faster from the rest in case of either success or failure. The three versions are:

- A simple reinforcement learning algorithm, in which the updating of the values is done through a direct decrement of the confidence value $C$ in the time $t$, according to the absolute value of the difference between the prediction $P$ and the real value $R$. The update of the values is expressed by Eq. (1).

$$
C_{t+1}=C_{t}-\mid(R-P) /
$$

- The revised Roth-Erev reinforcement learning algorithm [27] that, besides the features of the pre- vious algorithm, also includes a weight value $W$ for the definition of the importance of past experi- ence, which can be defined by the user. This ver- sion is expressed as in Eq. (2).

$$
C_{t+1}=C_{t} \times W-/(R-P) / \times(1-W)(2)
$$

- A learning algorithm based on the Bayes theorem of probability $[30,51]$, in which the updating of the values is done through the propagation of the probability of each algorithm being successful given the facts of its past performance. The ex- pected utility, or expected success of each algo- rithm is given by Eq. (3), being $E$ the available ev-idences, $A$ an action with possible outcomes $\mathrm{Oi}$, $U(O i \mid A)$ the utility of each of the outcome states given that action $A$ is taken, $P(O i \mid E, A)$ the con-

ditional probability distribution over the possible outcome states, given that evidence $E$ is observed and action $A$ taken.

$$
E U(A \mid E)=-\underset{i}{P(O i \mid E, A) \times U(O i \mid A)(3)}
$$

\subsubsection{Context analysis}

Contexts are an important factor in what concerns the adaptation of the approaches to be chosen as the final action to be performed in the market by the sup- ported player. A mechanism to analyze and define dif- ferent market negotiating contexts is executed by the Main Agent, hence providing the means for the chosen actions to be adapted and chosen depending of the dif- ferent circumstances that are encountered at each mo- ment.

The context definition process takes into consideration some influential conditionings or characteristics that affect the prices [41], suchas:

- The market price for the period and day in matter;

- The amount of transacted power in the market;

- The wind intensity verified in that period of the day (this is important because it affects the production of wind plants, and therefore the total ne- gotiated amount of power);

- The type of the day (whether it is a working day or weekend; if it is a holiday, or a special situation day, e.g. a day of an important event, such as an important game in a certain sport, which af- fects the energy consumption in that day, both be- cause of the consumption in the stadium, and for increasing the number of people with the TV on to watch it).

The grouping of a day's periods depending on their context is performed through the application of a clus- tering mechanism. The clustering mechanism analyses the characteristics of each period throughout the days, and attributes each period to the cluster that presents the most similar characteristics. The number of con- texts/number of clusters, can be defined by the user. It 
is also adapted by the Efficiency/Effectiveness balance management, so that a higher number of contexts is used when the available execution time is higher, and less contexts when the simulations have to be executed faster.

\subsubsection{Efficiency/effectiveness balance management}

The diversity of algorithms and approaches that are used by ALBidS bring out the need for the develop- ment of a mechanism that is able to manage the bal- ance between the Efficiency and Effectiveness (2E) of the system. This mechanism provides the means for the system to adapt its execution time to the purpose of the simulation, i.e., if the expected results from ALBidS are as best as it is able to achieve, or, on the other hand, if the main requirement is for the system to be exe- cuted rapidly, since the purpose of the considered simulation is to analyze issues other than player's optimal performance in the electricity market. For that the user can define a percentage value for preference of effi- ciency or effectiveness of the system. The 2E manage- ment mechanism manipulates the strategies both ex- ternally and internally. From the system's perspective this mechanism contributes by deciding which tools are used at each moment for each circumstance; depending on their observed performance in terms of ef- ficiency and effectiveness. This way this mechanism can choose to exclude certain strategies when they are not fulfilling the ALBidS' requirements for the case in matter. The strategies chosen to be executed are also manipulated internally, so that they can adapt their in- dividual results quality/execution time balance to the needs of each on-going simulation.

The adaptation process is performed by means of a fuzzy process $[53,63,66]$. Two dynamic fuzzy variables characterize the efficiency and the effectiveness of each strategy. The characterization is what concerns the efficiency of each strategy comprises the difference between each strategy's execution time, and the refer- ence execution time of the simulation without the use of decision support. This means that the higher the dif- ference is, i.e. the longer a strategy takes to achieve re- sults, when compared to the reference simulation time, a worse classification is attributed to the strategy. Regarding the characterization of the efficiency of each strategy, the quality of the forecasts is analyzed, com- paring the forecasted value, and the actual market price that was verified. The confusion matrix that combines the two fuzzy variables, plus the preference value of the user for a faster or better decision support, deter- mines which strategies must be excluded from the sys- 
tem, for taking too long to achieve not so good results, or which must adapt their execution times, re- ducing them in by a certain amount. The internal adap- tation of each strategy concerning the execution time is dependent on the characteristics of each strategy (e.g. a neural network can reduce the training data, to achieve faster, yet worse, results; the game theory strat- egy can reduce the number of considered scenarios; the optimization based strategies can use heuristic pro- cesses rather than deterministic approaches, in order to achieve faster results, even if only near-optimal).

\subsection{Strategy agents}

A highly dynamic environment such as the electric- ity market forces players to be equipped with tools that allow them to react to diverse negotiation circum- stances. The existence of a variety of different strate- gies grants ALBidS the capability of always being pre- pared for the diversity of situations that a market ne- gotiation player can encounter in the market. The very different natures of the considered strategies offer cov- erage over a diversity of areas, guaranteeing a high probability that is always one strategy suited for each different context, even if its applicability to other con- texts is not as advantageous. The considered strategies are:

- Based on statistical approaches:

- Average market prices of the same weekday for the last month;

- Average market prices of the last week considering only business days;

- Average market prices of the last four months;

- Regression on the market prices of the last four months;

- Regression on the market prices of the last five business days.

These are simple, yet very fast approaches, which are especially useful for cases when the execution time is critical.

- Dynamic Feed Forward Neural Network (NN) $[43,59]$ trained with the historic market prices, with an input layer of eight units, regard-ing the prices and powers of the same period of the previous day, and the same week days of the previous three weeks. The intermediate hidden layer has four units and the output has one unit - the predicted market price for the period in ques- tion. This $\mathrm{NN}$ is retrained in each iteration so that the data observed at each moment is considered for the next forecasts, this way constantly adapt-ing the NN forecasting results [43]. 
- Adaptation of the AMES bidding strategy. This strategy uses the Roth-Erev [27] reinforcement learning algorithm to choose the best among a set of possible bids that are calculated based on the relation cost/profit that the player presents when producing electricity. The various possible bids differ from each other due to the distinct combina- tion of the input parameters. The most combina- tions we set, the best chances there are of getting a good result. However, the number of combina- tions affects the processing time and the number of runs required for a satisfactory convergence. Complete details concerning the methodology of this strategy can be found in [33].

- The Composed Goal Directed strategy is based on two consecutive objectives, the first one may be increasing the profit, and the second one reduc- ing the greenhouse effect emissions. This strategy will try to obtain the highest profit, decreasing the price if in the same period of the previous day the first objective was not completely satisfied, and then try to fulfil the second goal, while maintain- ing the first satisfied.

- The Adapted Derivative-Following strategy is based on a Derivative Following strategy proposed by Greenwald [22]. The Adapted Derivative-Following strategy adjusts its price by looking at the amount of revenue earned in the same period of the previous day, as a result of that pe- riod's price change. If that period's price change produced more revenue per good than the same period of two days before, then the strategy makes a similar change in price. If the previous change produced less revenue per good, then the strategy makes a different price change.

- The Market Price Following strategy, as the name suggests, follows the market price of the same period of the previous day. It is a very simple strategy, but it presents good results when prices show a tendency to stabilize in a certain period, for some consecutive days.

- The SA-QL strategy [45] uses the Simulated Annealing heuristic [9] to accelerate the process of convergence of the Q-Learning [28] algorithm in choosing the most appropriate from a set of differ- ent possible bids to be used by the market nego- tiating agent whose behaviour is being supported by ALBidS.

- The Game Theory strategy is characterized as a scenario analysis algorithm able to support strate- gic behaviour, based on the application of the game theory $[40,42]$. This strategy creates several scenarios that represent different possibilities of the reality, concerning competitor players' actions and market environment itself. By analysing these scenarios and the possible actions that the sup-ported player can perform, a decision method ap- plied to choose the best, or the most safe, action to take, given the expected environment.

- The Economic Analysis strategy implements an analysis based on the two most commonly used approaches of forecasting in a company's scope. These approaches are the internal data analysis of the company, and the external, or sectorial, data analysis [46]. These two analyses, plus the risk as- sociated to the player's action, determine the ac- tion that should be performed.

- The Determinism Theory strategy executes a strategy based on the principles of the Determin- ism Theory [10]. This theory states that due to the laws of cause and effect, which apply to the mate- rial universe, all future events are predetermined.

- The Error Theory strategy's goal is to analyse the forecasting errors' evolution of a certain forecast- ing method [50], to try finding patterns in that er-ror sequence and provide a prediction on the next error, which will be used to adequate the initial forecast.

- A Support Vector Machine (SVM) [32] based strategy is used to forecast the electricity market prices, providing results with a similar quality to the NN, but requiring only half of the resources and execution time.

- The Metalearner strategies use the results of the learning process from all the other strategies presented before, as inputs to apply their own learning [44], and therefore create new outputs. ALBidS includes three versions of metalearners:

- The Simple Metalearner performs a simple ensemble average of the output values of all ALBidS' strategies, to create its output;

- The Weighted Metalearner includes a methodology in which the way the other strategies' outputs are considered for building the metalearner's output depends on their confidence weight to the Main Agent. This means that the better a strategy is performing, the higher its in-fluence on this method's results will be. This is done through the application of a weighted av- erage of the outputs of all strategies, using their 
confidence values in each context as weights; 
- The ANN based Metalearner uses a dynamic ANN to combine the outputs of the strategies,

using their associated confidence values as im- portance weights;

- The Six Thinking Hats [11] Metalearner performs an adaptation of a strategy for conflict

resolution in a meeting environment. The Six Thinking Hats methodology suggests attributing different roles (ways of thinking) for each intervenient, which are then used by a central entity (blue hat) to determine the solution to the problem. The representation of this method in ALBidS is performed by using different strate- gies as intervenient players, whose actions are combined by applying genetic algorithms [25].

All strategies allow the definition of their particular parameters, or can alternatively be executed with their default inputs (a combination of parameters for each strategy that has achieved good results for differ-ent simulations).

\subsection{Player profile definition}

In order to build suitable profiles of competitor agents, it is essential to provide players with strategies capable of dealing with the possible changes in com- petitors' behavior, allowing adaptation to their actions and reactions. For that, it is necessary to have adequate techniques to analyze the data properly, namely the his- toric of competitor agents past actions. Analogously to the definition of market operation strategies, the way each agent's bid is predicted can be approached in sev- eral ways. So, the way to deal with this issue was to fol- low the same idea as for the main system's methodol- ogy. There are several algorithms for defining the play- ers' profiles, all providing their predictions, and on top of that a reinforcement learning algorithm that chooses the one that is more likely to present the best answer according to the past experience of their responses for each particular market context.

The used reinforcement algorithm is the RothErev algorithm [46]. It presents a distinct set of statistics for each acting agent, for their actions to be predicted in- dependently from each other, and also for each mar- ket context. This means that an algorithm that may be presenting good results for a certain agent in a certain context, with its output chosen more often when bid- ding in this context, may possibly never be chosen as the output for another context or another player.

The update of the stats is done accordingly to the difference between the predictions and the action each 


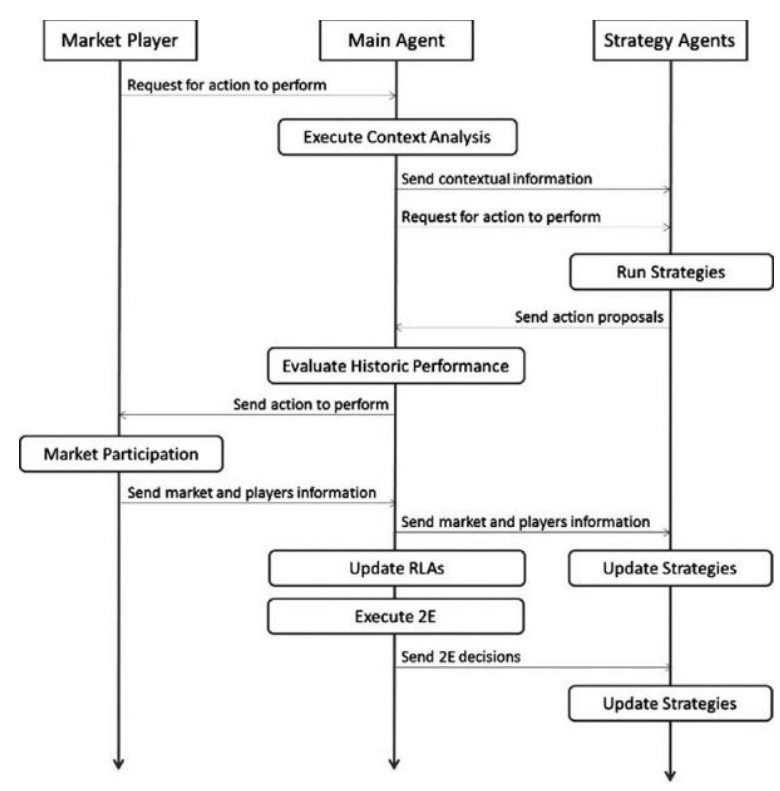

Fig. 2. Interactions between the involved agents.

player actually performed. The best rewards are attributed to the profile definition algorithms that present the smaller difference. This way, all algorithms' confi- dence values are updated in every prediction, whether a prediction is chosen as the final answer or not.

The strategies that are used to predict an agent's be- havior depend on the efficiency/effectiveness balance which is defined. If the preference is fully attributed to the efficiency of the method, only the faster strategies are used, allowing a huge reduction of the execution time. Otherwise, all strategies are used. However, the NN is adapted for each circumstance. The higher the preference for effectiveness, the bigger the amount of data considered for training the NN. This allows reduc- ing the execution time when required, even if just for a small amount, or increasing the quality of the predic- tions, when that is the main goal.

Figure 2 presents a sequence diagram, which shows the timings of the interactions between the involved agents, and how these interactions influence the execu- tion of the several methodologies. This figure refers to a single iteration (process for the decision support of a market player in a single participation in the electricity market).

From Fig. 2 it is visible that the decision support process starts with a request from the market partici- pant player, asking for the action it should perform in the market. When the Main Agent receives this request, the context analysis mechanism is executed, and the re- spective contextual information is sent to each strategy 
agent. A request for each Strategy Agent is also sent, so that each provides its action suggestion for the current context.

After receiving the request from the Main Agent, all Strategy Agents execute their respective methodologies, and send their individual action suggestion for the Main Agent. After receiving all proposals from the Strategy Agents, the Main Agent evaluates the historic performance of all strategies in the current context, and chooses the suggestion of the strategy that presents the higher confidence values for the current context as the final proposal for the supported market player. This fi- nal action proposal is sent to the market player.

The supported market player participates in the elec- tricity market using the proposed action, and after the market negotiations are terminated, the player sends the feedback to the Main Agent, providing all the in- formation concerning the market environment that it has encountered, including the information regarding the actions of the competitor players that have partici- pated in the same market session. The Main Agent for- wards this information to all Strategy Agents, so that they can update their individual strategies according to facts that have been observed in the market. While the Strategy Agents perform the update of their strategies, the Main Agent updates the RLAs, by comparing the suggestions that each Strategy Agent has provided with the actual market development. A new confidence value for each strategy for the current context is stored. The Main Agent also executes the $2 \mathrm{E}$ balance manage- ment mechanism, using the performance evaluation of each strategy, and the execution time that each Strat- egy Agent has needed to send its action proposal. The results of the 2E mechanism are sent to the Strategy Agents, and these perform once again their updating process, which, this time, refers to the reduction of the execution time, or to the finalization of the agent, in case its execution time is found to be irremediably high for the quality of results it is originating.

This process is performed once per iteration, i.e. ev- ery time the supported market player wishes to partic- ipate in the electricity market.

\section{Case study}

\subsection{Market negotiations' specification}

The spot or day-ahead market is a daily basis functioning market [36], where players negotiate electric power for each hour, or half hour of the following

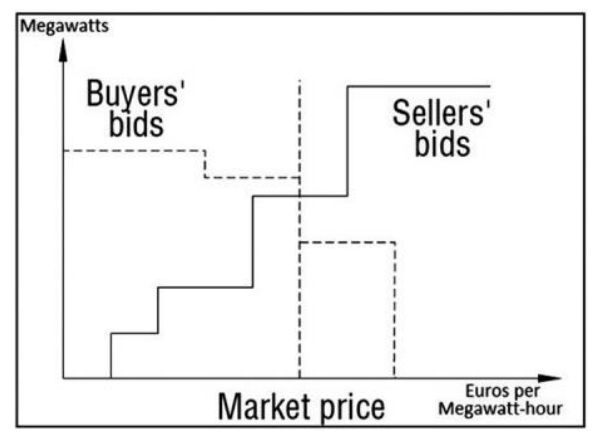

Fig. 3. Symmetric market price establishment.

day. Such markets are structured to consider production fluctuations as well as differences in production costs of distinct units.

In this market, each participating entity must present their selling or buying proposals for each of the 24 hourly periods of a day. These proposals or bids are typically composed by a tuple (power, price), with different meanings, whether they come from buyers or sellers, respectively: power stands for amount of power to be bought or sold, and price is the maximum ac- cepted price or minimum selling price.

When the negotiation is finished, an economic dis- patch for each period is set by the market operator. At the end of each period the market operator uses a market-clearing tool establishing the market price - a unique price that will be applied to all transactions of this period.

In market pools, the most common type of negotia- tion is a standard uniform auction. MIBEL day-ahead spot market works as a symmetric market, where both suppliers and consumers submit bids. The market oper- ator orders the selling and demand offers: selling bids start with the lowest price and move up, and demand bids start with the highest price and move down. Then, the proposed bids form the supply and demand step curves, and the point at which both curves intersect determines the market price, paid to all accepted sup- plier and consumers. The bids of every supplier offer-ing prices lower than the established market price and every consumer offering prices higher than the market price will be accepted. Figure 3 shows the symmetric market prices definition.

The profits can be improved by submitting bids that are advantageous for the player in the bidding process;

i.e. for a seller player, a bid price below the estab- lished market price, but still as high as possible, in or-der to assist in increasing the market price (origination of higher profits, through a higher 
market price). In the 
case of a buyer agent, the bid price should be above the established market price, but as low as possible, in or-der to reduce the cost that is paid for the bought energy.

\subsection{Specifications}

This section presents the results of a set of simulations undertaken using MASCEM, with the objective of assessing the performance of ALBidS, by comparing its performance to that of all the individual strate- gies that have been mentioned in this paper.

The metric for comparing the performance of the methods is the profits that each is able to originate for an electricity market participant player - a seller; since the goal of ALBidS and of all strategies is to maximize the profits of a market player. The costs of production are kept constant throughout all hours of all consid- ered days, in order to facilitate the comparison of the achieved profits.

In order to provide a suitable comparison, the same market scenario, with the exact same players, under the same circumstances, is executed repeatedly. The only variation is the behavior of the test subject player, Seller 2. In each simulation Seller 2 uses the decision support of each of the 20 mentioned strategies, and fi- nally of ALBidS, in order to compare the performance of all.

The simulations refer to the same 62 consecutive days (two months), starting from Saturday, $1^{\text {st }}$ De- cember, 2012, until Thursday, $31^{\text {st }}$ January, 2013. The data used in this case study has been based on real data extracted from the Iberian market operator-MI-

BEL [37], using an automatic data extraction that has been presented in [49].

This scenario was created with the intention of rep- resenting the Iberian reality, reduced to a smaller sum- marized group, containing the essential aspects of dif- ferent parts of the market, in order to allow a better individual analysis and study of the interactions and potentiality of each of those actors. Further details on the test scenario and on the specifications for this case study can be consulted in [13].

All comparisons are performed for three distinct cases: (i) $100 \%$ preference for the effectiveness of the strategies, i.e. all strategies, and consequently ALBidS, perform at their full potential; (ii) $50 \%$ preference for effectiveness, i.e. the execution times of the most de- manding strategies are reduced, which means a reduc- tion in their quality of results; (iii) $0 \%$ preference for the effectiveness, i.e., most of the strategies are ex-cluded from the system, while only the faster to exe- cute are maintained.
For all three cases, the context analysis mechanism receives the input of 4 , as the number of alternative contexts to be used, so that it is possible to compare the performance of the strategies when acting in differ- ent contexts. For the considered scenario, the four dif- ferent contexts are easy to understand: separation be- tween business days and weekends plus holidays; and separation from peak and off-peak consumption hours of the day. From the 62 considered days, 42 are busi- ness days and 20 are not ( 9 weekends, which equals 18 days, plustwo holidays verified in both countries of MIBEL (Portugal and Spain): $25^{\text {th }}$ December, and $1^{\text {st }}$ January). From the 24 hours of the day, 5 are grouped as peak hours of consumption: from $19 \mathrm{~h}$ to $23 \mathrm{~h}$; and the remaining 19 are clustered as off-peak. Therefore, the four different contexts are as follows:

- Context 1: Peak hours of business days (total of 210 periods during the 62 days);

- Context 2: Off-peak hours of business days (total of 798 hourly periods);

- Context 3: Peak hours of non-business days (total of 100 periods);

- Context 4: Off-peak hours of non-business days (total of 380 periods).

Besides the comparison of the profits that each strat- egy originates in each context, the strategies' confi- dence weights evolution throughout the time is also compared, as well as the rate each strategy is chosen as the final output of ALBidS. The choice process is undertaken using the Roth-Erev RLA (Eq. (2)), with a weight value $W$ for past events of 0,4 ; a low value to allow a faster adaptation to new observed events.

\subsection{Results for $100 \%$ preference for effectiveness}

Figure 4 presents the comparison of the confidence weights of the Main Agent on each of the strategies, throughout the simulation time, for each of the four contexts.

From Fig. 4 it is visible that, by starting with the same confidence weights and with no previous learning process, strategies take a number of iterations be- tween a significant separation can be observed. Dur- ing the first iterations the same strategies have pre- sented higher confidence weights in all contexts: the simpler strategies, with reduced or null learning capa- bilities, such as the strategies based on averages and regressions, and the simple metalearner. After a few it- erations, the SVM starts increasing its confidence val- ues, as this 
methodology requires a reduced amount 


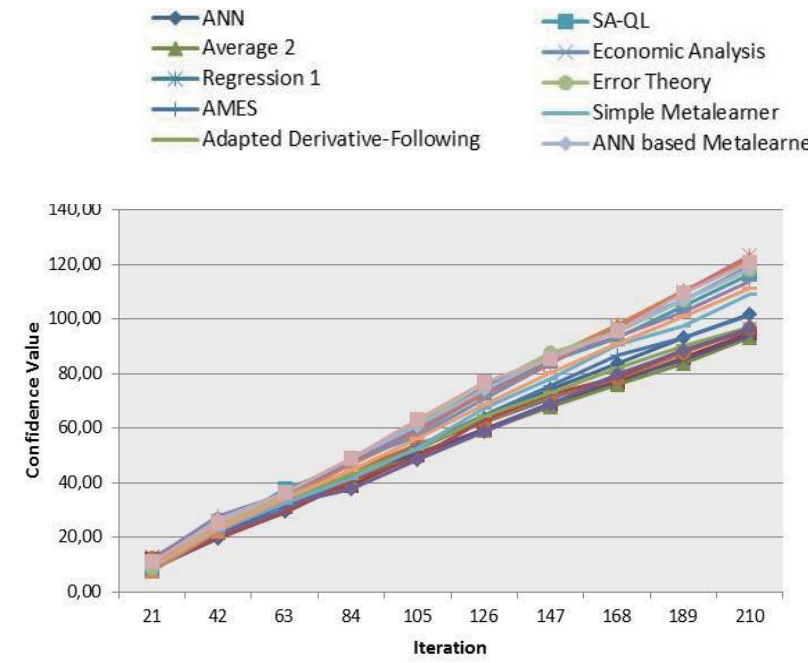

(a)

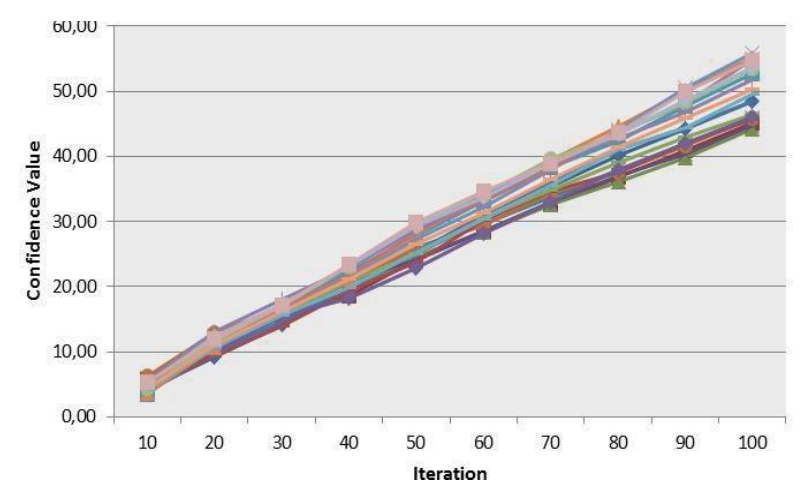

(c)

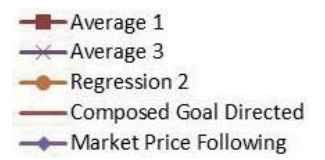

- -Game Theory

Determinism Theory

$\longrightarrow$ SVM

Weighted Metalearner

- - STH Metalearner

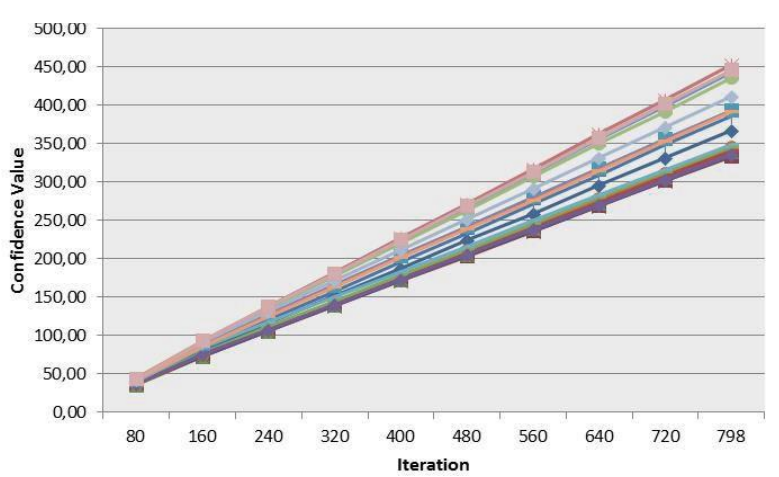

(b)

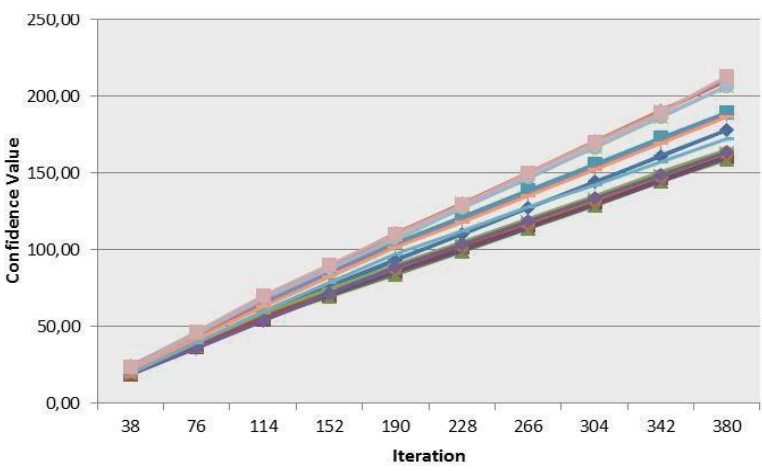

(d)

Fig. 4. Confidence weights of each of the strategies in: (a) Context 1, (b) Context 2, (c) Context 3, (d) Context 4.

of training data. As the time progresses, the strategies with more complex learning processes start improv- ing their performance, due to the experience that they start gathering, and their learning process starts be- coming more effective. In the final iterations of Con- text 3, in Fig. 4(c), which maximum number of itera- tions is 100 , it is visible that the more complete strate- gies start detaching from the simpler ones. This de- tachment is more evident from Fig. 4(a), in Context 1, which, with its 210 iterations allows the learning pro- cess of the most complete strategies to show better performances, therefore increasing their confidence values. From the Contexts with the higher number of iterations, namely Context 2 and Context 4, in Figs 4(b) and (d) respectively, not only is this detachment even more clear, as one can additionally see some intermedi- ate sub-groups, of medium complexity strategies, such as the SA-QL, the ANN-based metalearner, and the AMES strategy. In these contexts the simpler strate- gies show that their best confidence values during the first iterations are long gone, and they show the worst confidence values in the end. The group of strategies that achieves the higher confidence values in the bigger number of iterations is composed by the Game The- ory strategy, the Determinism Theory, The Economic Analysis, and the STH Metalearner.

Figure 5 presents the rate in which each strategy has been chosen by the Main Agent as the final output of ALBidS, in each context.

From Fig. 5 it is visible that the simpler strategies, such as the ones based on averages and regressions of the market prices, have been chosen a number of times in all 4 contexts. They have been chosen during the first iterations, while the other, more complex strategies do not reach an adequate learning maturity which enables them to achieve the most advantage results. The SVM has also been chosen a considerable amount of times in all four contexts (The most evident is in Context 3 - Fig. 5(c), which by presenting a smaller number of iter- ations, does not provide enough time for the most com- 


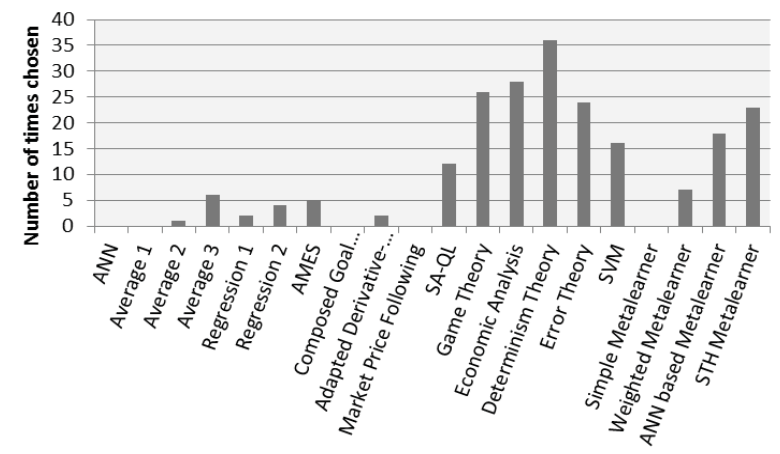

(a)

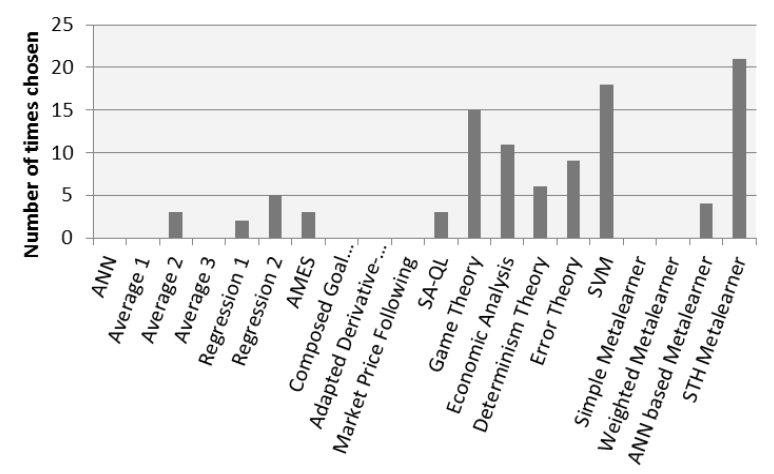

(c)

Fig. 5. Strategies choice rate for: (a) Context 1, (b) Context 2, (c) Context 3, (d) Context 4.

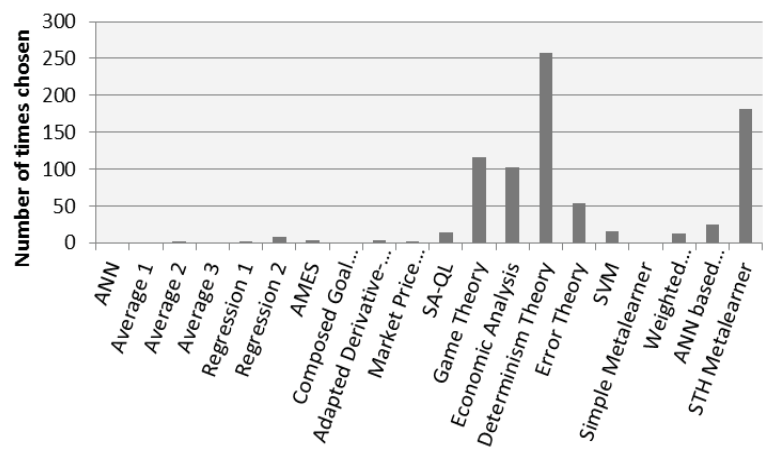

(b)

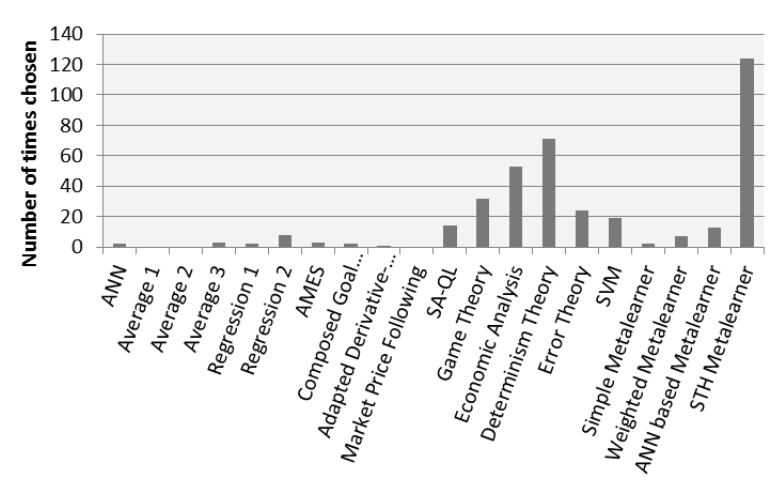

(d) plex strategies to evidence themselves, therefore the SVM still manages to end up as the second more cho- sen strategy). This is due to the low amount of train- ing data that this approach requires, which enables it to achieve good results from an early point. Intermediate complexity strategies such as the SA-QL, AMES, and the Weighted Metalearner, also present some amount of selections. However, it is evident that the strategies with the more complex learning capabilities, such as the Determinism Theory, the STH Metalearner, Game Theory, Economic Analysis, are the ones that end up being chosen more often, mainly in the contexts with the higher number of iterations, due to the best performance that is achieved after the learning process ma- tures.

Figure 6 presents the comparison of the profits that each strategy has provided for the supported market player in the total of the iterations of each context.

From Fig. 6 it is visible that ALBidS is able to achieve higher profits than all strategies, in all four contexts. The difference between ALBidS and the individual strategies is more visible in the contexts with larger number of iterations, namely Context 2 and 4, Figs $6(\mathrm{~b})$ and (d) respectively. The larger number of iterations gives more time for all strategies to refine their independent learning process, and ALBidS benefits from that, as the quality of choices improves. The contexts with the least number of iterations, namely Context 3, Fig. 6(c) represent a more balanced out- come between all strategies, although the difference between the quality of the strategies can still be no- ticed. Nevertheless, ALBidS is able to achieve higher profits than all. The good response of ALBidS in all contexts is supported by Fig. 7, which shows the total profits that have been achieved by each strategy in the total of the 24 periods of the 62 considered days (total of the four contexts).

From Fig. 7 it is visible that ALBidS has been able to provide higher profits for the supported player than all the other strategies, in the total of the 62 consid- ered days. The simpler strategies show that their ca- pability of outperforming the more complex strategies in the first iterations is not nearly enough to compete with those, as the differences are evident in the total profits. The strategies that show the best performances, and that obtain the higher profits are the Determinism Theory, followed very closely by the STH Metalearner, and by the Game Theory, Economic Analysis, and Er-ror Theory. 


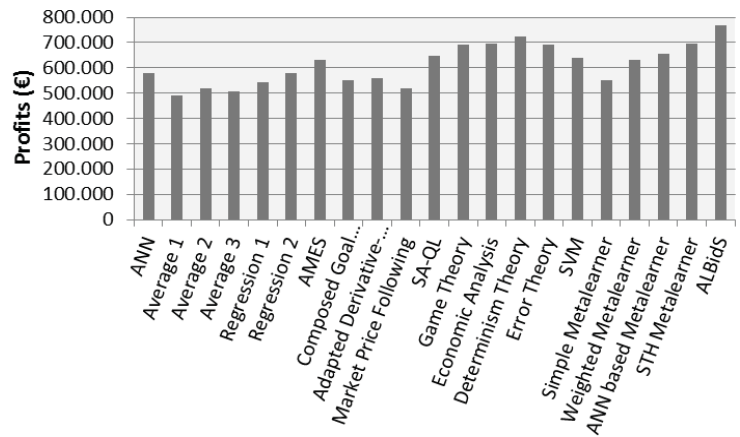

(a)

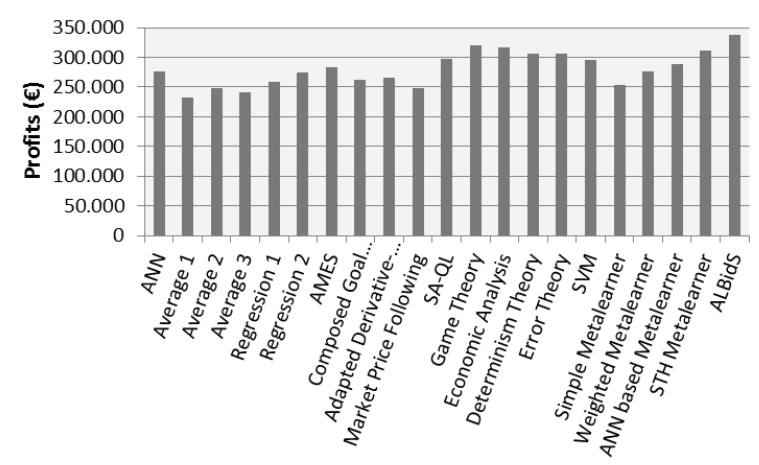

(c)

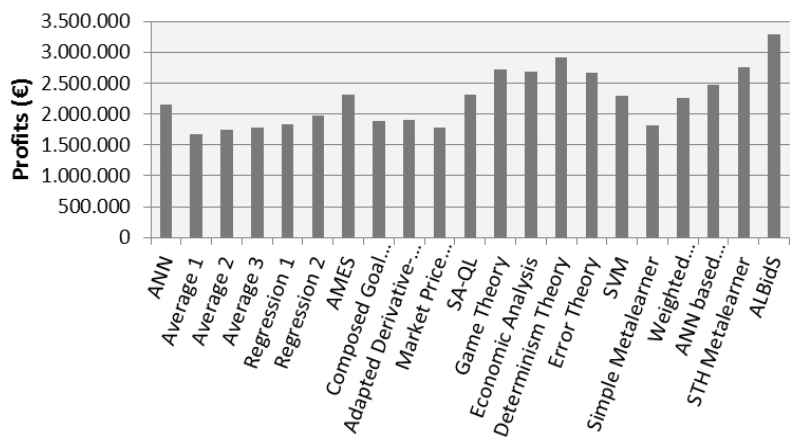

(b)

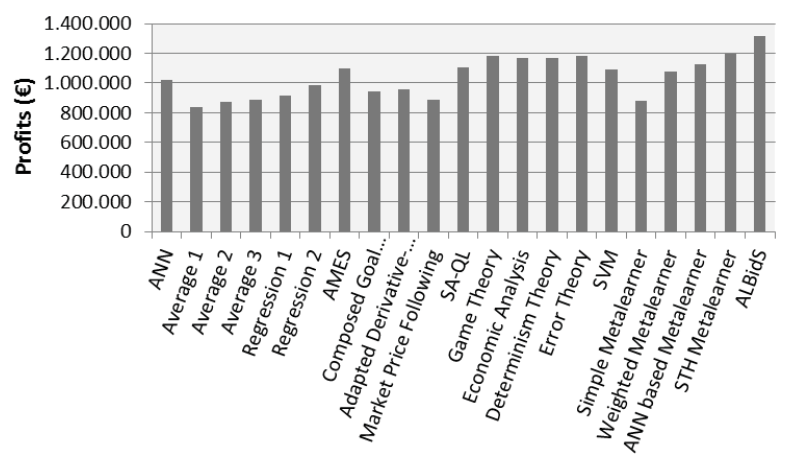

(d)

Fig. 6. Profits provided by all strategies, and by ALBidS, in: (a) Context 1, (b) Context 2, (c) Context 3, (d) Context 4.

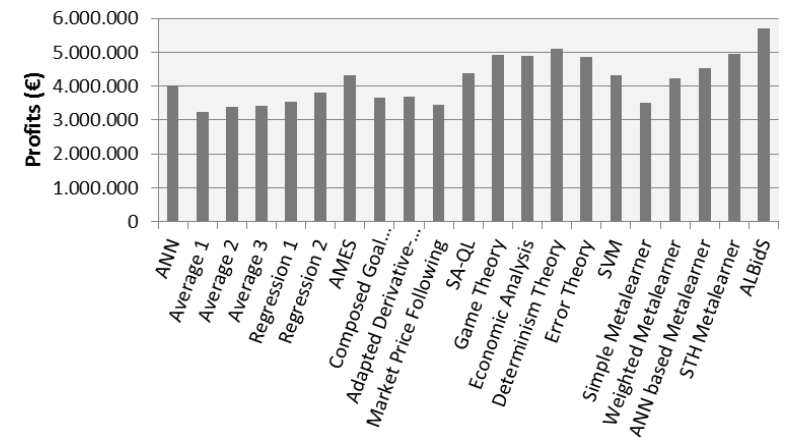

Fig. 7. Profits provided by all strategies, and by ALBidS in the total of the 62 considered days.

\subsection{Results for $50 \%$ preference for effectiveness}

The execution with $50 \%$ preference for effectiveness results in the reduction of the execution time of the most time demanding strategies. The ones that suffer the most from this reduction, which is verified by their decrease in quality of results, are the Game Theory strategy, the Determinism Theory, STH Metalearner. From the strategies that have achieved the best results in the case with $100 \%$ preference for effectiveness, the one that is required to reduce the execution time by a smaller amount, due to its relatively faster execution time when compared to the other more complex strate- gies, is the Economic Analysis. Table 1 presents a sum- mary of the results that have been verified in this case. From Table 1 it is visible that, despite the decrease in execution time, and consequent degradation in exe- cution time, the Determinism Theory strategy has still been able to be the strategy with the higher confi- dence value and the most chosen strategy in Context 1. In the other contexts, the Economic Analysis has been the strategy that achieves the best results. It is also visible that, while the simpler strategies maintain their performance, the most complex ones, present a decrease in their confidence values, when compared to the case with $100 \%$ preference for effectiveness. Figure 8 presents the profits that each strategy, and ALBidS have achieved in this case, in the total of the 62 days, for the four contexts.

From Fig. 8 it is visible that the strategies that presented the best results in the case with $100 \%$ preference for effectiveness have decreased their achieved prof- its. The exception is the Economic Analysis strategy, which is now the strategy that achieves the higher prof- its. ALBidS is once again able to achieve higher prof- 

Table 1

Summary of the case with $50 \%$ for effectiveness

\begin{tabular}{|c|c|c|c|c|c|c|c|c|}
\hline \multirow[b]{2}{*}{ Context } & \multicolumn{4}{|c|}{ Chosen rate } & \multicolumn{4}{|c|}{ Final confidencevalue } \\
\hline & 1 & 2 & 3 & 4 & 1 & 2 & 3 & 4 \\
\hline ANN & 0 & 0 & 0 & 2 & 101.92 & 367.56 & 48.44 & 178.0 \\
\hline Average 1 & 0 & 0 & 0 & 0 & 94.98 & 333.49 & 45.15 & 161.1 \\
\hline Average 2 & 1 & 2 & 3 & 0 & 93.45 & 340.48 & 44.27 & 159.0 \\
\hline Average 3 & $\overline{6}$ & 0 & 0 & 3 & 94.27 & 333.95 & 44.74 & $\overline{1} 60.4$ \\
\hline Regression 1 & 2 & 2 & 2 & 2 & 96.27 & 339.91 & 45.92 & 163.1 \\
\hline Regression 2 & 4 & 8 & 5 & 8 & 96.85 & 343.86 & 45.88 & 162.8 \\
\hline AMES & 5 & 4 & 3 & 3 & 101.65 & 385.64 & 48.50 & $\overline{1} 77.8$ \\
\hline Composed goal & 0 & 0 & 0 & 2 & 96.00 & 339.6 & 45.62 & 165.0 \\
\hline Adapted derivative- & 2 & & & & 97.31 & 2160 & 1616 & \\
\hline Märket price following & 0 & & & & 96.70 & & & \\
\hline SA-QL & 22 & 14 & 6 & 14 & 116.57 & 393.19 & 52.70 & 189.5 \\
\hline Game theory & 12 & 48 & 8 & 18 & 112.26 & 409.26 & 50.75 & 193.4 \\
\hline Economic analysis & 36 & 375 & 28 & 16 & 119.53 & 443.14 & 55.91 & 206.8 \\
\hline Determinism theory & 51 & 102 & 6 & 31 & 121.06 & 430.19 & 52.16 & 199.7 \\
\hline Error theory & 24 & 53 & 9 & 24 & 108.63 & 400.91 & 49.24 & $\overline{1} 89.9$ \\
\hline SVM & 16 & 26 & 18 & 38 & 113.91 & 393.21 & 51.84 & $\overline{1} 87.7$ \\
\hline Simple metalearner & 0 & & 0 & & 97.43 & -340.7 & -46.95 & 165.0 \\
\hline Weighted metalearner & 7 & 12 & 0 & 7 & 103.58 & 363.6 & 47.80 & 173.1 \\
\hline ANN based & 6 & 25 & 4 & 13 & 110.34 & & 49.91 & $\overline{192.1}$ \\
\hline STH metalearner & 16 & 123 & 8 & 46 & 112.45 & 414.2 & 50.94 & 198.0 \\
\hline
\end{tabular}

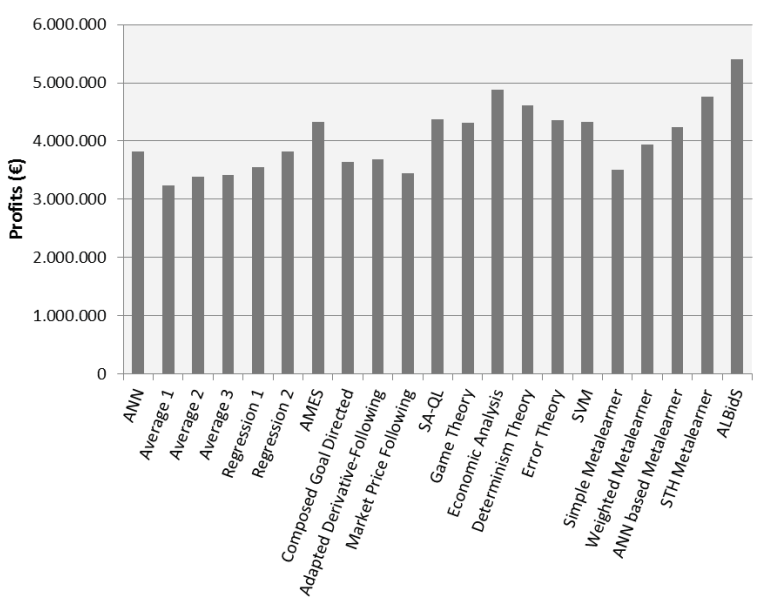

Fig. 8. Profits provided by all strategies, and by ALBidS in the total of the 62 considered days.

its than all strategies, by choosing the most appropriate strategy as the time progresses.

\subsection{Results for $0 \%$ preference for effectiveness}

Using $0 \%$ preference for the effectiveness of ALBidS means that all strategies that need more time than the execution time of MASCEM for running the market simulation, are excluded. This results in the uti- lization of a reduced number of strategies, i.e. only the faster to execute. Table 2 shows the summary of the re- sults for the case with $0 \%$ preference for effectiveness.

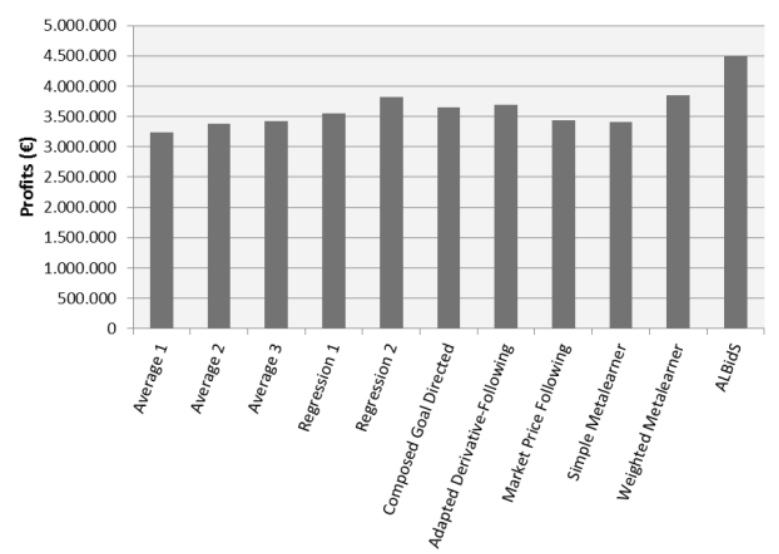

Fig. 9. Profits provided by all strategies, and by ALBidS in the total of the 62 considered days.

From Table 2 it is visible that using a reduced number of strategies leads to higher competitiveness between them. The faster response time and minor learn- ing capabilities from the strategies supports this fact. In the first two contexts, the strategy that was chosen more often was the Weighted Metalearner. In Context 3 the most chosen strategy was a regression approach, and in Context 4 , the Composed Goal Directed strat- egy. However, the Weighted Metalearner was the strat- egy that achieved the best confidence weight in the fi- nal of all iterations in all contexts except for one. Note that finishing the simulation with the higher confidence values does not necessarily mean that the strategy is chosen more often than others. It means that it is the 
Table 2

Summary of the case with $0 \%$ for

effectiveness

\begin{tabular}{|c|c|c|c|c|c|c|c|c|}
\hline \multirow[b]{2}{*}{ Context } & \multicolumn{4}{|c|}{ Chosen rate } & \multicolumn{4}{|c|}{ Final confidencevalue } \\
\hline & 1 & 2 & 3 & 4 & 1 & 2 & 3 & 4 \\
\hline $\begin{array}{l}\text { Average } 1 \\
\text { Average } 2\end{array}$ & $\begin{array}{l}0 \\
1\end{array}$ & $\begin{array}{r}4 \\
34\end{array}$ & $\begin{array}{l}0 \\
8\end{array}$ & $\begin{array}{l}6 \\
0\end{array}$ & $\begin{array}{l}94.98 \\
93.45\end{array}$ & $\begin{array}{l}333.4 \\
340.4\end{array}$ & $\begin{array}{l}45.15 \\
44.27\end{array}$ & $\begin{array}{l}161.1 \\
159.0\end{array}$ \\
\hline Average 3 & 6 & 8 & 0 & 3 & 94.27 & 333.9 & 44.74 & 160.4 \\
\hline Regression 1 & 14 & 29 & 6 & 6 & 96.27 & 339.9 & 45.92 & 163.1 \\
\hline Regression 2 & 31 & 82 & 34 & 8 & 96.85 & 343.8 & 45.88 & 162.8 \\
\hline Composed goal directed & 36 & 24 & 11 & 195 & 96.00 & $\overline{3} 39.6$ & 45.62 & 165.0 \\
\hline Adapted derivative- & 18 & 28 & 14 & 39 & 97.31 & 346.0 & 46.46 & 164.8 \\
\hline Märket pricefollowing & 11 & 8 & 4 & 6 & 96.70 & 335.2 & 46.02 & 164.0 \\
\hline Simple metalearner & 0 & 0 & 0 & 0 & 94.99 & $\overline{3} 37.0$ & 44.92 & 159.4 \\
\hline Weighted metalearn & 93 & 32 & 23 & 117 & 99.25 & 348.2 & 46.29 & 168.5 \\
\hline
\end{tabular}

one being chosen in the final iterations, but it also shown that different may be chosen much less often during previous iterations where its confidence value was not still as high. Fig- ure 9 presents the comparison of the achieved profits from the used strategies in the case with $0 \%$ preference for effectiveness.

From Fig. 9 it is visible that ALBidS has achieved higher profits than all the strategies, even when using a limited number of approaches. The Weighted Met- alearner was the strategy that achieved the higher prof- its, followed by Regression 2 .

\section{Conclusions}

This paper presented a model that enables choosing the most appropriate from a set of decision support strategies. This model takes into account the context in which strategies are being used, and uses reinforce- ment learning algorithms to update confidence values on each strategy, which facilitate the choice on the best strategy to be applied according to the previous perfor-mance that each has shown in each different context.

This methodology is the core of ALBidS, a multiagent system that provides decision support to electric- ity market negotiating players. ALBidS is integrated with the MASCEM simulator, which provides the means for testing and suiting ALBidS' implemented tools and mechanisms.

ALBidS' design, architecture, and integration with MASCEM proved to be adequately implemented, as showed by tests presented in Section 4. These exper- imental findings have shown that ALBidS is able to achieve higher profits for the supported market player than all of the implemented strategies. This has been verified for different contexts, and for cases where the used strategies are limited. The demonstration of re-sults for different contexts has 
strategies do, in fact, perform differently under differ- ent contexts. Although the best strategies tend to out- perform the others in all cases, they still show that the best strategy varies from context to context. The learn- ing capabilities of ALBidS are able to deal with that, showing an highly advantageous means of taking the best of the available assets (strategies) by understand- ing when and how they perform at their best.

The wide range of approaches that provide action proposals for the Main Agent to choose from (from mathematics to physics, from power systems' appli- cations to artificial intelligence techniques) guarantees that at each moment responses based different views are suggested, offering a high variety of possible ac- tions for the ALBidS system to consider. This provides the means for the system to increase its chances of al- ways be provided with at least one adequate answer, even when the majority of the approaches fail in the accomplishment of its purposes.

This fact is extended to the other mechanisms which complement the system, i.e. the Context Analysis mechanism, the Player Profile Definition mechanism, and the Efficiency/Effectiveness Management mecha- nism.

Finally, regarding the main entity in the ALBidS system - the Main Agent, it also proved to efficiently accomplish its purposes, showing its ability to choose the most appropriate actions for the supported player to take, by using the reinforcement learning algorithms at its disposal, and taking advantage on the complemen- tary mechanisms that aim to enlarge ALBidS' capabilities of adaptation and providing of intelligent decision support.

Among the many developments that ALBidS creation potentiated, allowing many future works and scientific findings, the continuous improvement of ALBidS considers the complementation of ALBidS with a new mechanism directed to the optimization 
of market players' investments in alternative markets. This can be done by analysing the characteristics and particularities of the several existing markets, includ- ing complementary markets such as derivatives mar- ket. This way, adequate predictions of the expected in- comes resulting from the investments in each of those markets can be achieved, depending on each circum- stance and context.

\section{Acknowledgments}

This work is supported by FEDER Funds through COMPETE program and by National Funds through FCT under the projects FCOMP-01-0124-FEDER: PEstOE/EEI/UI0760/2011, PTDC/SEN-ENR/122174 /2010, and SFRH/BD/80632/2011 (Tiago Pinto PhD).

\section{References}

[1] H. Adeli and S.L. Hung, Machine learning neural networks, genetic algorithms, and fuzzy systems, John Wiley and Sons, New York, 1993.

[2] H. Adeli and X. Jiang, Neuro-fuzzy logic model for freeway work zone capacity estimation, Journal of Transportation En- gineering 129(5) (2003), 484-493.

[3] H. Adeli and H.S. Park, Neurocomputing for design automa- tion, CRC Press, Boca Raton, Florida, 1995.

[4] S.K. Aggarwal, L.M. Sainia and A. Kumar, Dayahead price forecasting in ontario electricity market using variable- segmented support vector machine-based model, Electric Power Components and Systems 37 (2009), 495-516.

[5] F. Azevedo, Z. Vale, P.B. Oliveira and H.M. Khodr, A long- term risk management tool for electricity markets using swarm intelligence, Electric Power Systems Research 80 (Apr 2010), 380-389.

[6] F. Azevedo, Z. Vale and P. Oliveira, A decisionsupport sys- tem based on particle swarm optimization for multi-period hedging in electricity markets, IEEE Transactions on Power Systems 22(3) (August 2007), 995-1003.

[7] R. Badawy, A. Yassine, A. Heßler, B. Hirsch and S. Albayrak, A novel multi-agent system utilizing quantum-inspired evo- lution for demand side management in the future smart grid, Integrated Computer-Aided Engineering 20(2) (2013), 127141.

[8] P. Bajpai and S.N. Singh, Fuzzy adaptive particle swarm op- timization for bidding strategy in uniform price spot market power systems, IEEE Transactions on Power Systems 22(4) (2007), 2152-2160.

[9] S. Bandyopadhyay, Multiobjective simulated annealing for fuzzy clustering with stability and validity, IEEE Transactions on Systems, Man, and Cybernetics, Part C: Applications and Reviews 41(5) (2011), 682-691.

[10] B. Berofsky, Determinism, Princeton University Press, 1971. [11] E. de Bono, Six thinking hats, England: Penguin Books, 1985. [12] Y. Boutalis, M. Christodoulou and D. Theodoridis, Indirect adaptive control of nonlinear systems based on bilinear neurofuzzy approximation, International Journal of Neural Systems 23(5) (2013), 1350022. 
[13] Case Study details http://www.gecad.isep.ipp.pt/mascem/up loaded_files/case_studies/Case_Study_ALBidS _IC-AE.pdf.

[14] J. Chen and T. Wu, A computational intelligence optimiza- tion algorithm: Cloud drops algorithm, Integrated Computer- Aided Engineering, IOS Press 21(2) (2014), 177-188.

[15] S. Cincotti and G. Gallo, Genoa artificial power-exchange, Agents Artificial Intelligence (2013).

[16] A.K. David and F. Wen, Strategic bidding in competitive elec- tricity markets: A literature survey, in: Proc IEEE Power Eng Soc Summer Meeting 4 (Jul 2000), 2168-2173.

[17] S. Distefano and A. Puliafito, Information dependability in distributed systems: The dependable distributed storage system, Integrated Computer-Aided Engineering, IOS Press 21(1) (2014), 3-18.

[18] D. Feng, Z. Yan, J. Stergaard, Z. Xu, D. Gan, J. Zhong, N. Zhang and T. Dai, Simulation embedded artificial intelligence search method for supplier trading portfolio decision, let Generation Transmission \& Distribution 4 (Feb 2010), 221-230.

[19] V. Figueiredo, F. Rodrigues, Z. Vale and J.B. Gouveia, An electric energy consumer characterization framework based on data mining techniques, IEEE Transactions on Power Sys- tems 20(2) (2005).

[20] A.J. Fougères and E. Ostrosi, Fuzzy agentbased approach for consensual design synthesis in product configuration, Inte- grated Computer-Aided Engineering 20(3) (2013), 259-274.

[21] E. Freeman, B. Bates, K. Sierra and E. Robson, Head first de- sign patterns, O'Reilly Media; 1 edition, (4 November 2004),

[22] A. Greenwald, Shopbots and pricebots, Proceedings of the Sixteenth International Joint Conference on Artificial Intelli- gence IJCAI, Stockholm, 1999.

[23] J.O. Gutierrez-Garcia and K.M. Sim, Agentbased cloud workflow execution, Integrated Computer-Aided Engineering 19(1) (2012), 3956.

[24] S. Harp, S. Brignone, B.F. Wollenberg and T. Samad, Sepia: A simulator for electric power industry agents, IEEE Control Systems Magazine 20(4) (2000), 53-69.

[25] L. Jia, Y. Wang and L. Fan, Multiobjective bilevel optimiza- tion for production-distribution planning problems using hy- brid genetic algorithm, Integrated Computer-Aided Engineer-ing, IOS Press 21(1) (2014), 77-90.

[26] X. Jiang and H. Adeli, Dynamic fuzzy wavelet neuroemulator for nonlinear control of irregular highrise building structures,
International Journal for Numerical Methods in Engineering 74(7) (2008), 1045-1066.

[27] Z. Jing, H.W. Ngan, Y.P. Wang, Y. Zhang and J.H. Wang, Study on the convergence property of re learning model in electricity market simulation, Advances in Power System Con- trol, Operation and Management (2009),

[28] C. Juang and C. Lu, Ant colony optimization incorporated with fuzzy q-learning for reinforcement fuzzy control, IEEE Transactions on Systems, Man and Cybernetics, Part A: Systems and Humans 39(3) (2009), 597-608.

[29] V.S. Kodogiannis, M. Amina and I. Petrounias, A clustering- based fuzzy-wavelet neural network model for short-term load forecasting, International Journal of Neural Systems 23(5) (2013), 1350024.

[30] K. Korb and A. Nicholson, Bayesian artificial intelligence, Chapman \& Hall/CRC, 2003.

[31] V. Koritarov, Real-world market representation with agents: Modeling the electricity market as a complex adaptive system with an agent-based approach, IEEE Power \& Energy maga- zine, (2004), 39-46.

[32] D. Li, L. Xu, E. Goodman, Y. Xu and Y. Wu, Integrating a statistical background-foreground extraction algorithm and 
SVM classifier for pedestrian detection and tracking, Inte-grated Computer-Aided Engineering 20(3) (2013), 201-216.

[33] H. Li and L. Tesfatsion, Development of open source software for power market research: The AMES test bed, Journal of Energy Markets 2(2) (2009), 111-128.

[34] F. Liu and M.J. Er, A novel efficient learning algorithm for self-generating fuzzy neural network with applications, Inter- national Journal of Neural Systems 22(1) (2012), 21-35.

[35] L. Meeus, K. Purchala and R. Belmans, Development of the internal electricity market in Europe, The Electricity Journal 18(6) (2005), 25-35.

[36] MIBEL - Operador del Mercado Ibérico de Energia, home- page. Available: http://www.omel.es/.Last accessed (January 2014), .

[37] MIBEL data files http://www.omie.es/aplicaciones/datosftp/ datosftp.jsp?path=/.

[38] G. Migliavacca, SREMS-electricity market simulator based

Game Theory and incorporating network constraints, IEEE Power Tech, Lausanne, Swiss (2007).

[39] M. Shafie-khah, M.P. Moghaddam and M.K. Sheikh-El- Eslam, Price forecasting of day-ahead electricity markets us- ing a hybrid forecast method, Energy Conversion and Man-agement 52 (May 2011), 2165-2169.

[40] J. Neumann, The essence of game theory,

IEEE Potentials 22(2) (2003).

[41] T. Pinto, Adaptive learning in agents behaviour: A framework for electricity markets simulation, Dissertation to obtain the Master of Science Degree in Computer Science - Special- ization in Knowledge-Based and Decision Support Technolo- gies, ISEP-IPP (2011),

[42] T. Pinto, I. Praca, Z. Vale, H. Morais and T.M. Sousa, Strate- gic bidding in electricity markets: An agent-based simulator with game theory for scenario analysis, Integrated Computer- Aided Engineering, IOS Press pág 20(4) (September 2013), 335-346.

[43] T. Pinto, H. Morais, P. Oliveira, Z. Vale, I. Praça and $C$. Ramos, A new approach for multi-agent coalition formation and management in the scope of electricity markets, Energy 36(8) (2011), 5004-5015.

[44] T. Pinto, T.M. Sousa, Z. Vale, I. Praca and H. Morais, Met- alearning in ALBidS: A strategic bidding system for electric- ity markets, Highlights on Practical Applications of Agents and Multi-Agent Systems - Advances in
Intelligent and Soft Computing pág. J. Pérez, M. Sánchez, P. Mathieu et al., eds, Springer Berlin Heidelberg 156 (2012), 247-256.

[45] T. Pinto, T.M. Sousa, Z. Vale, H. Morais and I. Praça, Intel- ligent decision making in electricity markets: Simulated an- nealing q-learning, IEEE Power and Energy Society General Meeting 2012, San Diego CA, USA, (22-26 July 2012),

[46] M. Porter, How competitive forces shape strategy, Harvard business Review, 1979.

[47] PowerTAC (http://www. powertac.org/).

[48] I. Praça, C. Ramos, Z. Vale and M. Cordeiro, MASCEM: A multi-agent system that simulates competitive electricity mar- kets, IEEE Intelligent Systems, Special Issue on Agents and 18(6) (Markets, 2003) 54-60.

[49] I. Praça, T.M. Sousa, A. Freitas, T. Pinto, Z. Vale and M. Silva, Adaptive tool for automatic data collection of real electricity markets, Intelligent Agent Technology in Electricity Markets and Power Systems (IATEM) workshop of the 23rd Interna- tional Conference on Database and Expert Systems Applica- tions - DEXA 2012, (3-6 September 2012).

[50] J. Principe, Information theoretic learning, Springer, Informa- 
tion Science and Statistics series, 2010.

[51] Z. Rabinovich, V. Naroditskiyb, E.H. Gerdingb and N.R. Jen- nings, Computing pure BayesianNash equilibria in games with finite actions and continuous types, Artificial Intelli-gence, Elsevier, (February 2013).

[52] C.W. Richter, G.B. Sheble and D. Ashlock, Comprehensive bidding strategies with genetic programming/finite state au- tomata, IEEE Trans on Power Systems 14(4) (1999), 12071212.

[53] G.G. Rigatos, Adaptive fuzzy control for differentially flat MIMO nonlinear dynamical systems, Integrated Computer- Aided Engineering, IOS Press 20(2) (2013), 111-126.

[54] C. Rodriguez and G. Anders, Energy price forecasting in the ontario competitive power system market, IEEE Transactions on Power Systems 19(1) (February 2004).

[55] E.J. Rodriguez-Seda, D.M. Stipanovic and M.W. Sponga, Teleoperation of multi-agent systems with nonuniform con- trol input delays, Integrated Computer-Aided Engineering 19(2) (2012), 125-136.

[56] G. Santos, I. Praca, T. Pinto, S. Ramos and Z. Vale, Scenarios generation for multi-agent simulation of electricity markets based on intelligent data analysis ieee symposium on intelli- gent agent (IA) at the IEEE SSCI 2013 (IEEE Symposium Se- ries on Computational Intelligence), Singapore, (15-19 April, 2013).

[57] M. Shahidehpour, H. Yamin and Z. Li, Market operations in electric power systems: Forecasting, scheduling, and risk management, Wiley-IEEE Press, (2002), 233274.

[58] S. Shamoun and D. Sarne, Increasing threshold search for best-valued agents, Artificial Intelligence, Elsevier, (June- July, 2013).

[59] N. Siddique and H. Adeli, Computational intelligence - syn- ergies of fuzzy logic, neural networks and evolutionary com- puting, Wiley, West Sussex, United Kingdom, 2013.

[60] P. Trigo, P. Marques and H. Coelho, (Virtual) Agents for run- ning electricity markets, Simulation Modelling Practice and Theory 18(10) (2010), 1442-1452.

[61] Z. Vale, Intelligent power system, in: Encyclopedia of Com- puter Science and Engineering, 5-Volume set (ISBN 978-0- 47138393-2), B.W. Wah, ed., pág., Wiley, Hoboken, NJ, 3 (January 2009), 1604-1613.

[62] Z. Vale, T. Pinto, I. Praça and H. Morais, MASCEM - Elec- tricity markets simulation with strategic players, IEEE Intel- ligent Systems 26(2) (Markets, 2011), 54-60 Special Issue on Al in Power Systems and Energy.

[63] E.I. Vlahogianni and M.G. Karlaftis, Fuzzy- entropy neural network freeway incident duration modeling with single and competing uncertainties, Computer-Aided Civil and Infrastructure Engineering 28(6) (2013), 420-433.

[64] D.D. Wu, D.X. Niu and D. Liu, A soft computing system for day-ahead electricity price forecasting, Applied Soft Comput-ing 10 (Jun 2010), 868-875.

[65] A.D. Yucekayaa, J. Valenzuelaa and G. Dozierb, Strategic bidding in electricity market using particle swarm optimiza- tion, Electric Power Systems Research 79 (2009), 335-345.

[66] Y. Zhang and H. Ge, Freeway travel time prediction using takagi-sugeno-kang fuzzy neural network, Computer-Aided Civil and Infrastructure Engineering 28(8) (2013), 594603.

[67] R. Zimmermann and R.J. Thomas, PowerWeb: A tool for evaluating economic and reliability impacts of electric power market designs, IEEE PES Power Systems Conference and Exposition 3 (2004), 1562-1567. 